\title{
Antibiotikatherapie
}

\section{S.-boulardii-Einnahme reduziert das Diarrhö-Risiko}

Gastrointestinale Nebenwirkungen sind eine häufige Folge einer antibiotischen Therapie. Diese können durch direkte Effekte auf den Gastrointestinaltrakt oder durch Störungen der intestinalen Flora verursacht werden [1].

\section{Darmflora wird geschützt}

Saccharomyces (S.) boulardii besitzt bei gleichzeitiger Einnahme mit einem Antibiotikum einen schützenden Effekt auf die Darmflora, da die medizinische Hefe zu einer Verminderung der Antibiotikaassoziierten Reduktion der Darmflora führt. Zudem fördert sie die Regeneration der Darmflora nach der Antibiotikagabe [2].
Wird die Darmflora durch Antibiotika geschädigt, so werden Ballaststoffe im Dickdarm nicht ausreichend abgebaut und weniger kurzkettige Fettsäuren gebildet, was zu Diarrhöen führt. S. boulardii erhöht den Gehalt an kurzkettigen Fettsäuren. Die Darmzellen können diese zusammen mit Natrium aufnehmen gleichzeitig wird passiv Wasser resorbiert und der Darminhalt eingedickt [3].

Eine Metaanalyse von fünf klinischen Studien mit zusammen mehr als 1.000 Patienten konnte zeigen, dass sich das Risiko einer Antibiotika-assozierten Diarrhö bei frühzeitiger und ausreichend hoch dosierter Einnahme von S.boulardii-haltigen Zubereitungen um mehr als $60 \%$ senken lässt [4]. Eine spätere prospektive Studie bestätigte diese Ergebnisse [5].

Diese Datenlage fand schließlich Niederschlag im Arzneimittelverordnungsreport 2013. Dort heißt es: Eine Metaanalyse dieser Studien erlaubt ... eine positive Einschätzung der Wirksamkeit von Probiotika zur Behandlung der Diarrhö. Auch bei Antibiotika-bedingten Nebenwirkungen scheint die zusätzliche Gabe von S. boulardii effektiv zu sein. - - Red.

- Literatur:

1. Fuhr R et al. Gastroenterologe. 2006;1:173-9

2. Swidsinski et al. Gastroenterology. 2013;144:824

3. Breves $G$ et al. Digestion. 2000;61:193-200

4. Szajewska H, Mrukowicz J. Aliment Pharmacol Ther. 2005;

22:365-72

5. Can M et al. Med Sci Monit. 2006;12:P119-22

\section{Nicht-valvuläres Vorhofflimmern}

\section{Schlaganfallprophylaxe mit gutem Nutzen-Risiko-Profil}

Bei den meisten Patienten mit nichtvalvulärem Vorhofflimmern (VHF) ist eine orale Antikoagulation zur Prophylaxe von Schlaganfällen unverzichtbar. Eine Therapie mit dem Faktor-Xa-Hemmer Apixaban (Eliquis ${ }^{\circledast}$ ) besitzt eine hohe Wirksamkeit bei geringem Blutungsrisiko und ist in der Praxis einfach zu handhaben. In mehreren klinischen Studien hat sich die Substanz hinsichtlich des Nutzen-Risiko-Profils mit am günstigsten erwiesen, sagte Prof. Martin Moser vom Universitäts-Herzzentrum Freiburg/Bad Krozingen.

\section{ARISTOTLE: Weniger Schlaganfälle, weniger schwere Blutungen}

In der Studie ARISTOTLE (Apixaban for Reduction in Stroke and other thromboembolic events in atrial fibrillation) bei 18.201 Risikopatienten mit nicht-valvulärem VHF war der FaktorXa-Hemmer (5 mg bzw. 2,5 mg, jeweils

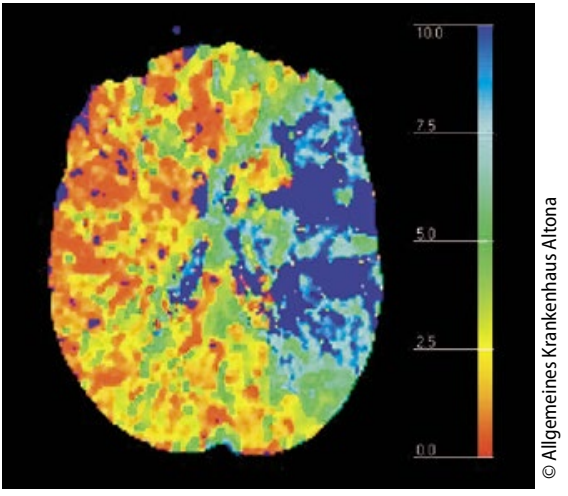

Bei Vorhofflimmern gilt es, einen Infarkt in der A. cerebri media (wie hier) zu verhindern.

zweimal täglich) in der Schlagfanfallprophylaxe signifikant wirksamer als der Vitamin-K-Antagonist Warfarin. Das Risiko für Schlaganfälle oder systemische Embolien (primärer Endpunkt) war im Verlauf von im Median 1,8 Jahren in der Apixaban-Gruppe relativ um
$21 \%$ verringert (Hazard Ratio $0,69, \mathrm{p}=$ $0,01)$. Die Rate ischämischer Schlaganfälle wurde fast halbiert ( $0,24 \%$ vs. $0,47 \%$ pro Jahr, $\mathrm{p}<0,001$ ) und die Gesamtmortalität (sekundärer Endpunkt) relativ um $11 \%$ reduziert $(\mathrm{p}=0,047)$ [Granger CB et al. N Engl J Med. 2011].

Deutliche Vorteile im Vergleich zur Warfarin-Gruppe wurden auch beim Blutungsrisiko (sekundäre Endpunkte) dokumentiert. Das Risiko für schwere Blutungen war unter Apixaban relativ um $31 \%(\mathrm{p}<0,001)$, das Hirnblutungsrisiko sogar um mehr als die Hälfte verringert $(\mathrm{p}<0,001)$. Bei der Rate gastrointestinaler Blutungen gab es keine Unterschiede zwischen der Apixaban- und der Warfarin-Gruppe.

Roland Fath

- Pressegespräch „Apixaban in der Schlaganfallprophylaxe bei Vorhofflimmern - Sicherheits- und Wirksamkeitsaspekte in der Antikoagulation, Praxisfälle und aktuelle Studienergebnisse"; Wiesbaden, April 2015 (Veranstalter: Bristol-Myers Squibb und Pfizer) 